\title{
ARDS in Obese Patients: Specificities and Management
}

\author{
Audrey De Jong ${ }^{1,2}$, Daniel Verzilli ${ }^{1}$ and Samir Jaber ${ }^{1,2^{*}}$
}

\begin{abstract}
This article is one of ten reviews selected from the Annual Update in Intensive Care and Emergency Medicine 2019. Other selected articles can be found online at https://www.biomedcentral.com/collections/ annualupdate2019. Further information about the Annual Update in Intensive Care and Emergency Medicine is available from http://www.springer.com/ series/8901.
\end{abstract}

\section{Introduction}

Obesity is a global healthcare problem that has risen to epidemic proportions worldwide [1,2]. It is now common to admit obese medical or surgical patients to the intensive care unit (ICU) [3]. It is estimated that at least $20 \%$ of patients admitted to the ICU are obese $[4,5]$. One of the main challenges of the critical care management of obese patients is successful respiratory system management. The negative effects of thoracic wall weight and abdominal fat mass on pulmonary compliance, leading to decreased functional residual capacity and arterial oxygenation, are exacerbated by a supine position and further worsened after general anesthesia and mechanical ventilation. Obese patients are at risk of developing lung de-recruitment and then atelectasis. The incidence of acute respiratory distress syndrome (ARDS) is increased in obese patients [6,7].

However, while obesity contributes to many diseases and is often associated with higher all-cause mortality in the general (non-selected) population [1], obese patients with ARDS have a similar or lower mortality risk when compared with non-obese patients with ARDS [7-9]. Obese patients therefore represent a specific population of ICU patients, and could differ from non-obese

\footnotetext{
* Correspondence: s-jaber@chu-montpellier.fr

${ }^{1}$ PhyMedExp, University of Montpellier, INSERM U1046, CNRS UMR 9214, Montpellier, France

${ }^{2}$ Anesthesia and Critical Care Department B, Saint Eloi Teaching Hospital, Centre Hospitalier, Universitaire Montpellier, Montpellier, France
}

patients. The main aim of this chapter is to summarize the most recent data on the epidemiology, outcomes, pathophysiology, ventilatory support and adjuvant therapies of the obese patient with ARDS.

\section{Epidemiology and pathophysiology Obesity and risk of ARDS}

Obese patients are particularly at risk of ARDS. In 1795 patients admitted from 1997 to 2009 to two centers, Gong et al. [6] reported that obesity was associated with ARDS compared with normal weight (odds ratio [OR] 1.66 [95\% confidence interval (CI) 1.21-2.28] for obese; OR 1.78 [95\% CI 1.12-2.92] for severely obese). Higher body mass index (BMI) and obesity were associated with longer lengths of stay but not ARDS mortality after adjusting for baseline clinical factors. Similar to these results, in a secondary analysis from a prospective, multicenter, international cohort in 2004 of 4968 adult patients in 349 ICUs, Anzueto et al. [7] observed a higher incidence of ARDS and acute renal failure in obese patients. After adjustment, obesity was significantly associated with the development of ARDS with ORs of 1.69 (95\% CI 1.07-2.69) for obese and 2.38 (95\% CI 1.154.89) for severely obese compared with normal weight; however, there were no associations with increased duration of mechanical ventilation, length of stay or mortality. These results were confirmed in a recent meta-analysis [10], which showed that obesity was associated with a significantly increased risk of ARDS (pooled OR 1.89 [95\% CI 1.45-2.47], $\left.\mathrm{I}^{2}=50 \%, p<0.00001, n=30,583\right)$.

The respiratory physiology of the obese patient might explain the increased incidence of ARDS criteria in obese patients. Anatomic and physiological alterations are observed in obese patients, affecting the face, neck, pharynx, chest wall and lungs. Excess abdominal fat may increase abdominal pressure. The displacement of the diaphragm upward, added to the increased chest wall weight, may raise baseline pleural pressure [11]. While total lung capacity and spirometric values usually remain normal, there is a decrease in functional residual 
capacity. Reduced functional residual capacity can trigger the closure of peripheral dependent airways during tidal ventilation, and decreased lung compliance due to tidal ventilation below the lower inflection point of the inspiratory pressure-volume curve. These changes result in atelectasis and ventilation-perfusion mismatch and hypoxemia, these effects being increased in the supine position. These modifications pave the way for infections and associated ARDS. The increased prevalence of gastroesophageal reflux disease and difficult intubation [12] can also increase aspiration incidence during intubation, and the underlying insult of ARDS [13].

It is worth noting that despite the increased incidence of ARDS in obese patients, only two of the four prospective scores predicting lung injury, the Lung Injury Prediction Score (LIPS) [14] and the Emergency Department Lung Injury Prediction Score (EDLIPS) [15], include obesity as a prognostic factor. Obesity status is not included in the surgical lung injury prediction score (SLIP-2 score) [16] or the early acute lung injury score (EALI score) [17].

\section{Prognosis of obese patients with ARDS}

Although the prognosis of obese ARDS patients remains debated, it seems that obese patients with ARDS have a similar or better prognosis than non-obese patients. Table 1 shows the main observational studies that have reported epidemiology and outcome in obese patients with ARDS. Ni et al. [23] showed in a meta-analysis performed in ARDS patients that compared with normal weight, being underweight was associated with higher mortality (OR 1.59, 95\% CI $1.22-2.08, p=0.0006$ ), while obesity (OR $0.68,95 \% \mathrm{CI}$ $0.57-0.80, p<0.00001$ ) and morbid obesity (OR 0.72 , 95\% CI $0.56-0.93, p=0.01$ ) were more likely to result in lower mortality. These results were similar to those of another meta-analysis performed by Zhi et al. [10]: obesity was significantly associated with reduced risk of ARDS mortality. However, longer prognosis is more uncertain. In a recent analysis of 144 candidate predictors in a large, multicenter, prospective cohort of ARDS survivors, obesity at admission was associated with worse 6-month quality of life (EQ-5D) [24].

There are several potential explanations for these findings, although data delineating mechanisms are lacking. Obese patients may have been misclassified as ARDS in case of interpretation of atelectasis as bilateral infiltrates. Although Gong and colleagues [6] found that increased frequency of $\mathrm{PaO}_{2} / \mathrm{FiO}_{2}<200$ was the criterion responsible for the higher ARDS incidence in obese patients, rather than radiographic findings, atelectasis is also associated with a low $\mathrm{PaO}_{2} /$ $\mathrm{FiO}_{2}$ ratio [25]. Despite a similar pulmonary injury, obese patients might be more prone to hypoxemia because of a greater incidence of atelectasis, which could result in a lower $\mathrm{PaO}_{2} / \mathrm{FiO}_{2}$ ratio compared with normal weight patients, and therefore a misdiagnosis of ARDS. Reduced functional residual capacity could participate in atelectrauma in case of non-appropriate ventilatory settings (positive end-expiratory pressure [PEEP] too low), worsening hypoxemia. Furthermore, recent evidence suggests that obesity induces a low-grade inflammation, generating a process that may subsequently protect the lung against further insults, through a mechanism of pre-conditioning [4]. However, two important confounding factors limit the extrapolation of these observational studies and could explain their discrepancy. The type of obesity (grade, repartition of fat [android vs. gynoid], sarcopenic/ non-sarcopenic obesity) is not indicated and muscular strength and function and the metabolic status of the patients (insulin sensitivity) were not evaluated. Diaphragmatic force may also be stronger in obese patients, as recently suggested by an experimental study performed in obese Zucker rats [26]. In obese rats, the diaphragmatic force was increased at baseline and after mechanical ventilation, compared to non-obese rats, which might be a protective factor in case of ARDS onset, facilitating liberation from mechanical ventilation. Another key element when considering the relationship between obesity and ARDS is that clinicians might consider obese patients to be at high risk of worse outcome; this belief could result in earlier admission to the ICU. Increased monitoring, associated with an increased use of prophylactic measures, could then explain the better prognosis observed in obese patients [4]. The medical or surgical status of the patients could also be a confounding factor. Our team evaluated the impact of medical admission as opposed to surgical admission on the short and long term outcome of obese ICU patients, in a prospective, observational cohort study of 791 obese patients admitted over 14 years, using a propensity-score-matched analysis [27]. Twenty percent of the patients included had ARDS. The main results were that ICU mortality in the medical group was higher than that in the surgical group and remained significantly higher 365 days post-ICU admission. After adjustment for category of admission, ICU mortality did not differ in obese and non-obese patients [22]). The current notion that all obese patients have similar ICU outcomes should be reconsidered.

\section{Ventilator support}

A summary of the recommendations for ventilatory management of the obese patient with ARDS is given in Box 1. 


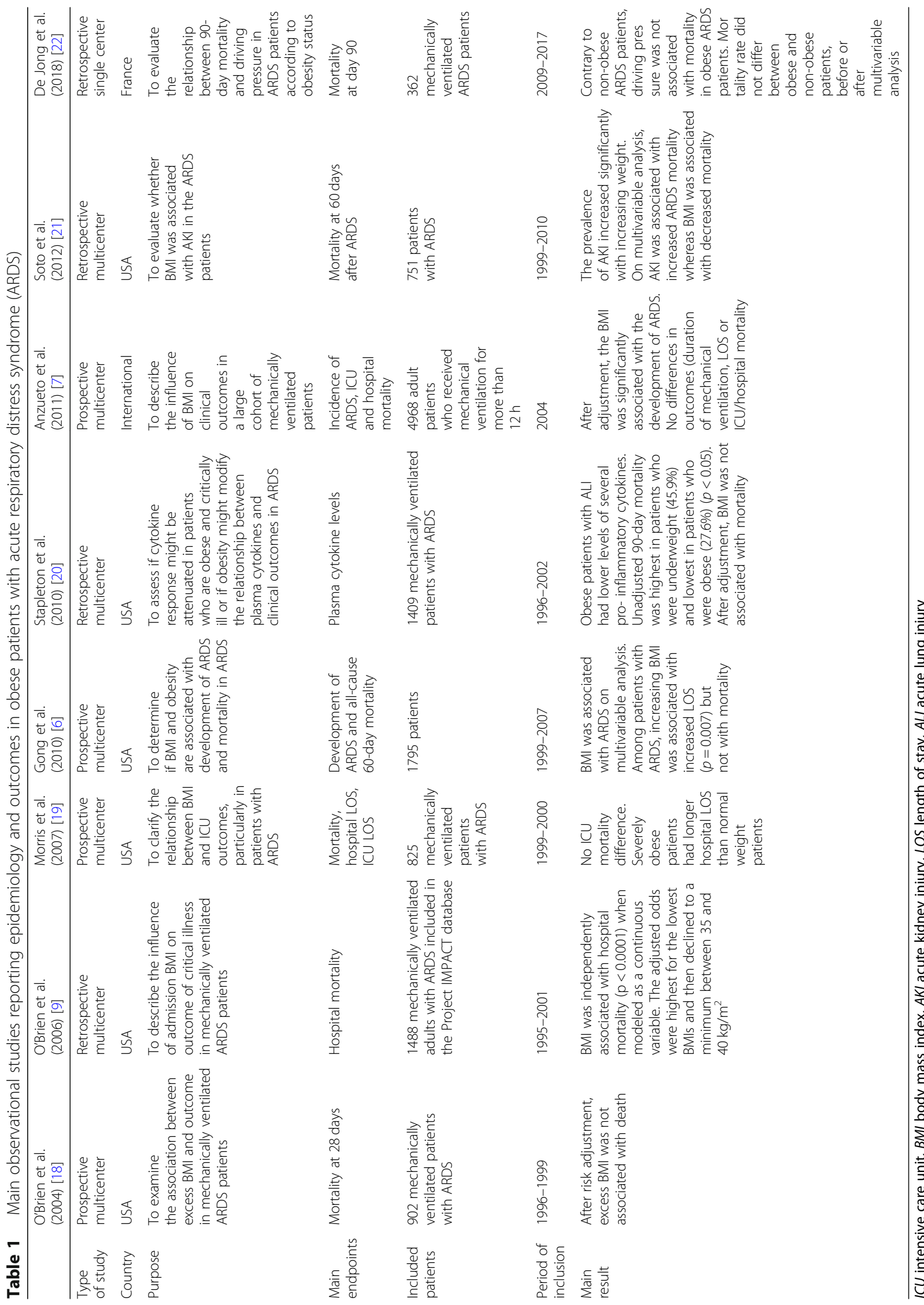




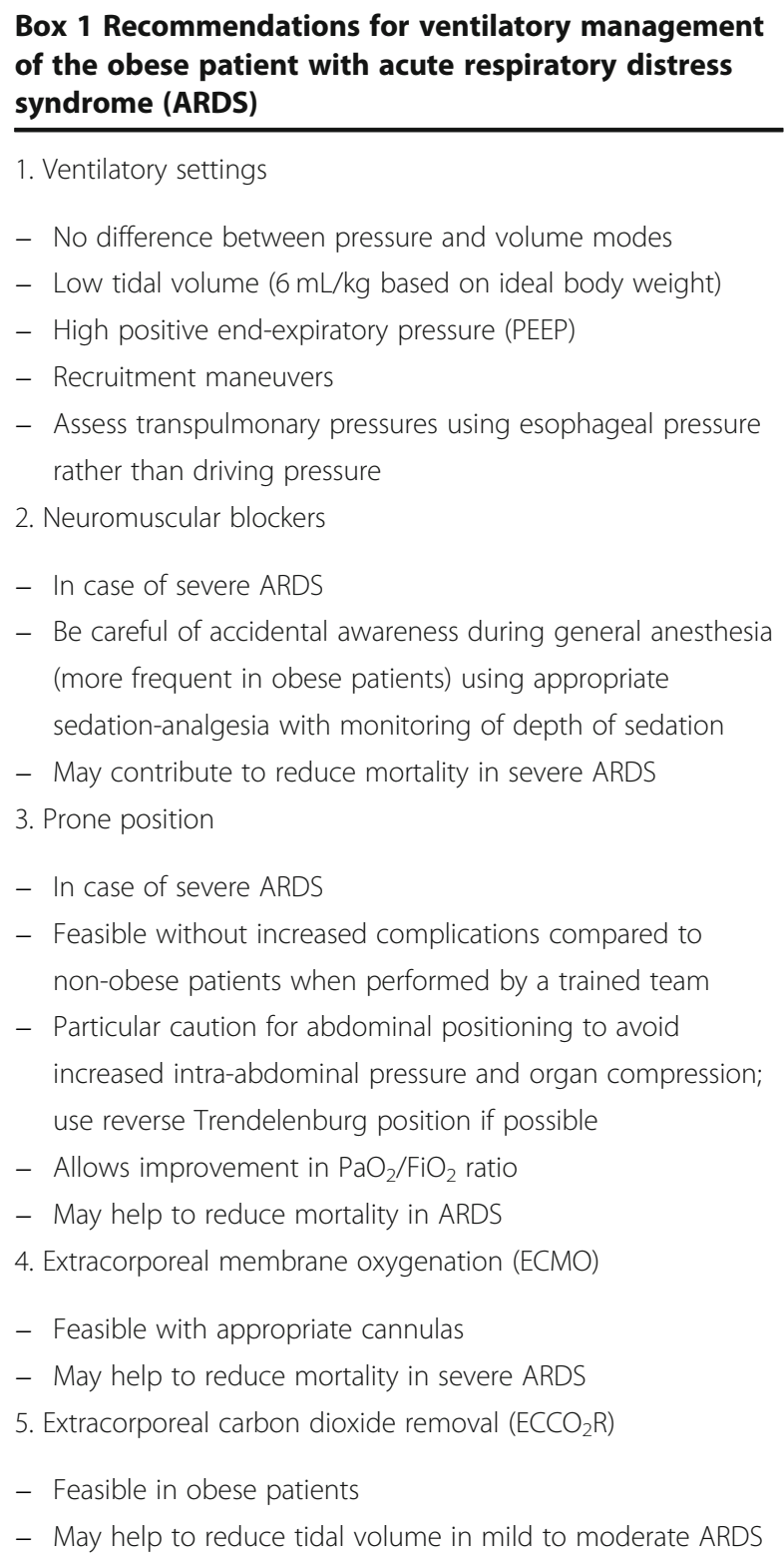

\section{Positioning}

A reverse Trendelenburg position, in which the supine patient's head is placed higher than the feet at an angle of $45^{\circ}$, might help to liberate the obese patient from the ventilator [28]. The reverse Trendelenburg position could act by reducing transdiaphragmatic pressure and atelectasis, resulting in improved gas exchange.

\section{Ventilator settings}

Protective ventilation should be applied, using low tidal volume, moderate to high PEEP, and recruitment maneuvers [29]. As the lungs do not grow with weight gain [3], tidal volume should be set according to ideal body weight, based on height and sex, and not actual body weight $[3,12]$. In the 2010s, these recommendations of low tidal volume according to ideal body weight were poorly applied [30]. In 580 mechanically ventilated adult patients admitted to three ICUs between February 1, 2006 and January 31, 2008, O'Brien et al. [30] reported that morbidly obese patients were often ventilated with significantly higher tidal volumes based on the predicted body weight compared with patients of normal body weight. Similarly, in the study by Anzueto et al. [7], a retrospective analysis of prospective data collected in 2004, severely obese patients were more likely to receive low tidal volumes based on actual body weight but high volumes based on predicted body weight. The ventilatory management of obese patients has probably changed since the periods of inclusion of these two studies. In the French center of Montpellier, we performed an analysis of ventilator settings in obese and non-obese ARDS patients from 2009 to 2017 [22]. Four hundred patients with ARDS were included, 295 non-obese patients and 105 obese patients. In contrast to the two previous studies $[7,30]$, we observed that ventilator settings were appropriate in obese and non-obese patients: tidal volume based on ideal body weight was not significantly different between obese and non-obese patients [22].

To take into account the increased abdominal pressure and chest wall mass and to limit atelectasis occurrence, higher PEEP is needed in obese patients compared to non-obese patients [31]. In a recent study, Pirrone et al. [32] showed that PEEP values commonly used by clinicians $\left(11.6 \pm 2.9 \mathrm{cmH}_{2} \mathrm{O}\right)$ was inadequate for optimal mechanical ventilation for morbidly obese ICU patients. A recruitment maneuver followed by end-expiratory pressure titration significantly improved lung volumes, respiratory system elastance and oxygenation. These authors assessed the effects of two approaches for titrating PEEP (a decremental PEEP trial and an end-expiratory transpulmonary pressure approach), and reported no significant differences on end-expiratory lung volume, respiratory mechanics or gas exchange. The two methods of evaluating PEEP identified the same optimal PEEP levels $\left(20.7 \pm 4.0\right.$ vs. $\left.21.3 \pm 3.8 \mathrm{cmH}_{2} \mathrm{O} ; p=0.40\right)$. Similar results were reported in obese patients in the operating room under general anesthesia in the study by Nestler et al. [33]. The authors [33] performed individualized PEEP titration using electrical impedance tomography and noted that a mean PEEP value of $18 \mathrm{cmH}_{2} \mathrm{O}$ was required to optimize end-expiratory lung volume. However, patients receiving optimized PEEP had greater need for intravenous fluids and vasopressors [33].

\section{Driving, Transpulmonary and transthoracic pressures}

Some studies have suggested that higher driving pressure (driving pressure $=$ plateau pressure $-\mathrm{PEEP})$ was associated with higher mortality in ARDS [34, 35]. However, the relationship between driving pressure and mortality 
has been poorly studied in obese patients with ARDS. The respiratory system (Fig. 1) includes the lung and the chest wall, and the airway pressure is related to both transpulmonary pressure (lung assessment, =alveolar pressure - pleural pressure) and transthoracic pressure (chest and abdomen assessment, = pleural pressure - atmospheric pressure), which differ in obese compared to the non-obese patients [12]. The relative part of pressure due to transthoracic pressure is higher in the obese patient than in the non-obese patient (elevated pleural pressure, which can be estimate by esophageal pressure) [36]. The key factor generating ventilator-induced lung injury (VILI) is regional lung overdistension with high transpulmonary pressure (Fig. 1). These lesions are usually apprehended by the evaluation of the plateau pressure, which represents the pressure used to distend the chest wall plus lungs. As seen earlier, obese patients have a very stiff chest wall, with elevated baseline pleural pressure, and much of the pressure that is applied by the ventilator will be used to distend the chest wall rather than the lung. An elevated plateau pressure may be related to an elevated transthoracic pressure, rather than an increase in transpulmonary pressure, with accompanying lung overdistension. We hypothesized that in obese patients with ARDS, with limited ventilated lung area and stiff chest wall, driving pressure would not be representative of the real pressure applied to the lungs and would not be associated with mortality [22]. We observed that the driving pressure at day 0 in non-obese patients was significantly lower in survivors at day 90 $\left(11.9 \pm 4.2 \mathrm{cmH}_{2} \mathrm{O}\right)$ than in non-survivors $(15.2 \pm 5.2$ $\left.\mathrm{cmH}_{2} \mathrm{O}, p<0.001\right)$. However, in obese patients, driving pressure at day 0 was not significantly different in survivors at day $90\left(13.7 \pm 4.5 \mathrm{cmH}_{2} \mathrm{O}\right)$ than in non-survivors $\left(13.2 \pm 5.1 \mathrm{cmH}_{2} \mathrm{O}, p=0.41\right)$. These results were confirmed in multivariable analysis, showing that driving pressure was not an independent factor for mortality in obese patients [22]. The results of this recent study suggest that driving pressure could not be appropriate to assess the severity or prognosis of obese patients with ARDS [22].

To differentiate the chest wall pressure from the lung pressure, assessing transpulmonary pressure using transesophageal pressure may be appropriate in these

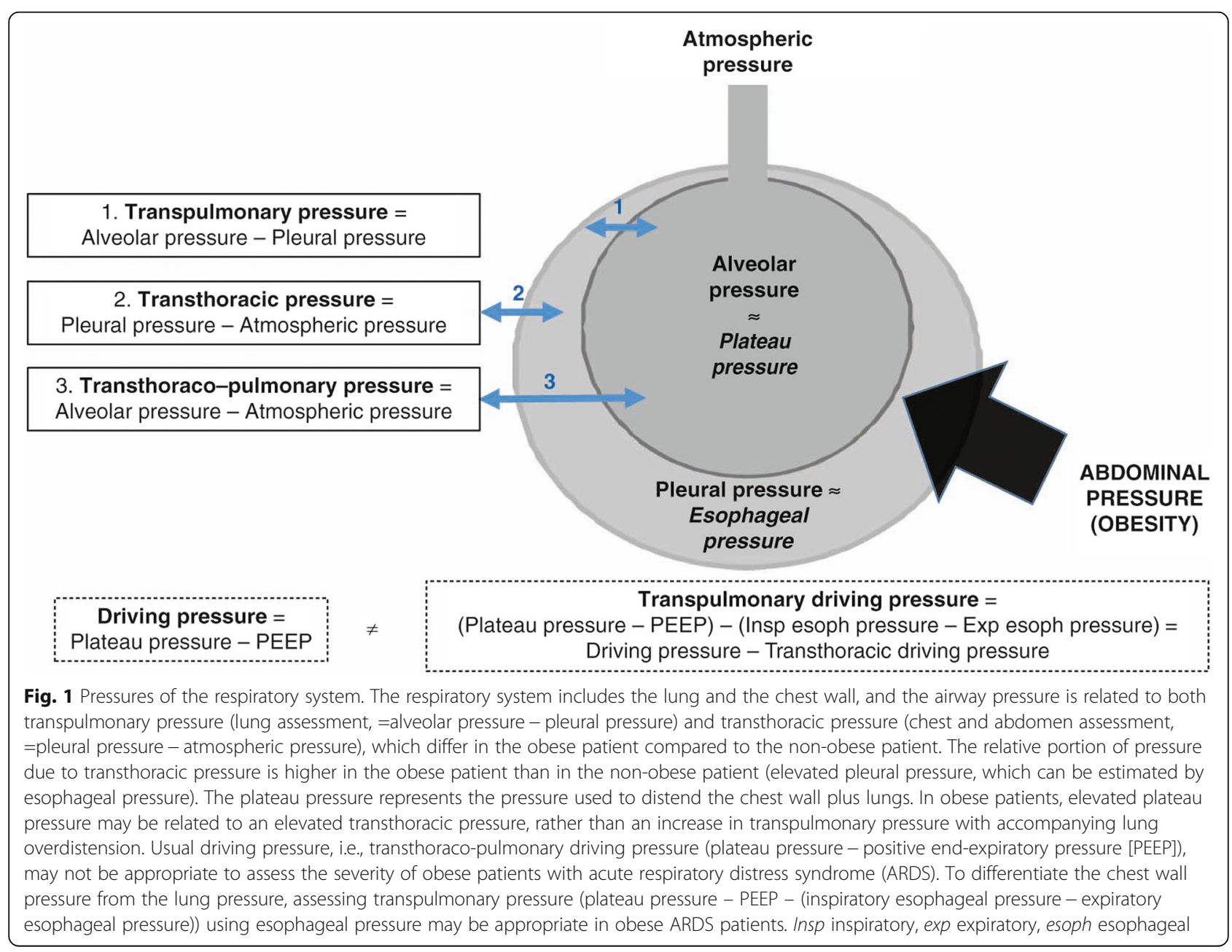


patients. A recent study examined the relationships between the respiratory system and transpulmonary driving pressure (the difference between airway plateau minus PEEP and inspiratory esophageal pressure minus expiratory esophageal pressure), pulmonary mechanics and 28-day mortality [37]. The results suggest that using PEEP titration to target positive transpulmonary pressure via esophageal manometry causes both improved elastance and driving pressures. Treatment strategies leading to decreased respiratory system and transpulmonary driving pressure at $24 \mathrm{~h}$ were associated with improved 28 day mortality. This strategy could be applied in obese patients, using esophageal pressure monitoring. Eichler et al. [38] showed that during laparoscopic bariatric surgery, patients require high peroperative levels of PEEP to maintain a positive transpulmonary pressure throughout the respiratory cycle. In the critical care setting, Fumagalli et al. [39] aimed to determine the relationship between transpulmonary pressure, lung mechanics, and lung morphology in obese patients with acute respiratory failure. They reported that, in obesity, low-to-negative values of transpulmonary pressure predict lung collapse and intratidal recruitment/ derecruitment. These results further support, for some authors, the use of transpulmonary pressure, using esophageal pressure monitoring, to monitor obese patients in ARDS.

\section{Neuromuscular blockers}

Neuromuscular blockers can be used in obese patients. When neuromuscular blockers are used, special care must be taken to prevent awareness during general anesthesia [40], because of rapid redistribution of sedatives in fat. Bispectral index (BIS) monitoring may help prevent accidental awareness during general anesthesia, although its efficacy for this purpose remains debated.

\section{Adjuvant therapies}

Prone position Prone positioning enables recruitment of more tissue in the dorsal region than can be derecruited in ventral regions, and lung inflation is more homogeneously distributed along the dorsoventral axis of the lung with a decrease in ventilation perfusion inequalities. In a non-obese population, Guérin et al. [41] showed that in patients with severe ARDS, early application of prolonged prone positioning sessions significantly decreased 28-day and 90-day mortality. As pointed out earlier, obese patients are particularly prone to atelectasis with a decreased functional residual capacity and may be more likely to respond to prone positioning. In a specific population of obese patients, the safety and efficiency of prone position in obese patients, defined using a BMI $>35 \mathrm{~kg} / \mathrm{m}^{2}$, were analyzed [8]. The primary endpoint was to evaluate the rate of complications of prone positioning and the secondary endpoint was to assess the effect on gas exchange, the nosocomial infection rate and mortality. For the 66 patients evaluated, at least one complication occurred in 20 patients, at similar rates in obese and non-obese patients (10/33 vs. 10/33, $p=1.00$ ). The $\mathrm{PaO}_{2} / \mathrm{FiO}_{2}$ ratio was significantly increased with prone compared to supine positioning in the two groups $(p<0.0001)$. In obese patients, the $\mathrm{PaO}_{2} /$ $\mathrm{FiO}_{2}$ ratio was significantly higher with prone positioning than in non-obese patients $(p=0.03)$, whereas there was no statistically significant difference for the supine position. This study was a single center study performed by a trained team. Particular care was needed to avoid increased abdominal pressure and associated complications, such as renal failure and hypoxic hepatitis [42], which might be triggered by prone positioning. To avoid these side-effects, the reverse Trendelenburg position and optimal abdominal fat positioning can be used. The technique is described in Fig. 2.

Extracorporeal membrane oxygenation The use of venovenous extracorporeal membrane oxygenation (VV-ECMO) has reemerged as an option for ARDS refractory to conventional support [43]. In addition to cannulation difficulty, obtaining sufficient circuit flow can be challenging in morbid obese patients [44]. There remains significant hesitancy in many centers to offer ECMO support to the obese population. However, class III obesity was not associated with poorer outcomes in obese ECMO patients in a recent study [45]. Among the 55 patients with ARDS placed on ECMO during the study period, 12 were morbidly obese $\left(B M I>40 \mathrm{~kg} / \mathrm{m}^{2}\right)$. Pre-ECMO mechanical ventilatory support and indices of disease severity were similar between the two groups, as were cannulation strategy and duration of ECMO support. Nine (75\%) morbidly obese patients and 27 (63\%) non-morbidly obese patients were successfully weaned from ECMO support, and patient survival to time of discharge was 67 and $58 \%$, respectively. In the subset of super obese patients (BMI $>50 \mathrm{~kg} / \mathrm{m}^{2}, n=6$ ), recovery and midterm survival was $100 \%$. Transport of morbidly obese patients receiving ECMO is also safe [46]. In the recent EOLIA study [47] performed in patients with very severe ARDS, including obese patients (only patients with BMI $>45 \mathrm{~kg} / \mathrm{m}^{2}$ were excluded), 60-day mortality was not significantly lower with ECMO than with a strategy of conventional mechanical ventilation that included ECMO as rescue therapy. However, $28 \%$ of the patients in the control group crossed over to ECMO for refractory hypoxemia. Based on these data, also reported by others [48], ECMO support in ARDS patients should not be withheld from the obese patient population. 


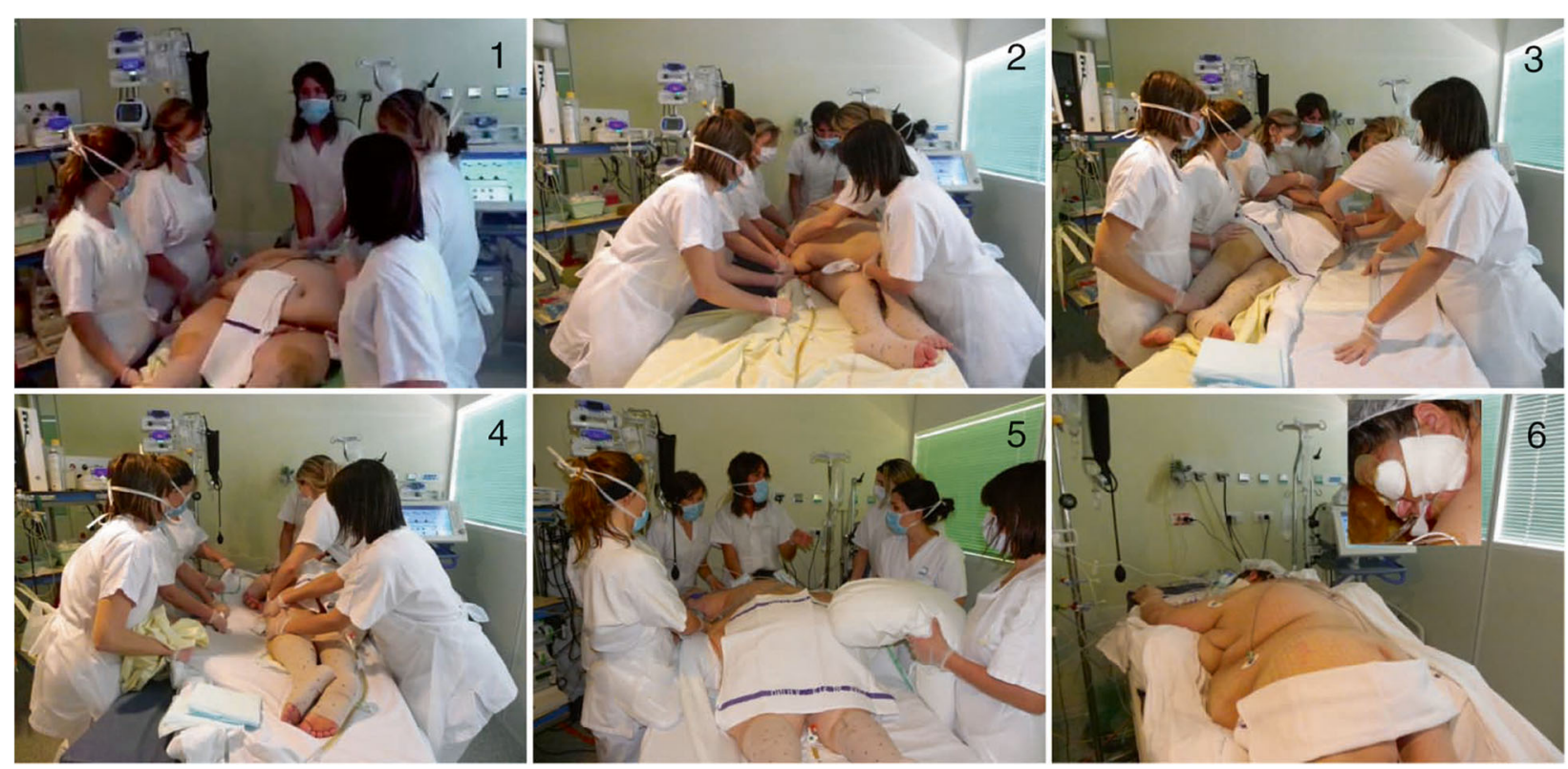

Fig. 2 Prone positioning of obese patients. Step 1: The patient is lying down, under deep sedation and analgesia. One operator is at the head of the patient to secure the airway access, three operators are on the right, two on the left, and one is mobile. Step 2: The monitor is checked. The patient is then turned on the left side first. Step 3: The patient is then moved to the other side of the bed. Step 4: The patient is turned. Step 5: Upper chest and pelvic supports are placed to avoid abdominal compression. Step 6: Finally, compression points are checked regularly, and the head is turned every $2 \mathrm{~h}$. Bed is positioned in a reverse Trendelenburg position

Extracorporeal carbon dioxide removal Use of extracorporeal carbon dioxide removal $\left(\mathrm{ECCO}_{2} \mathrm{R}\right)$ in non-obese and obese patients with ARDS is under evaluation [43, 49]. A recent pilot [50] study performed in 20 patients with mild to moderate ARDS and a mean BMI of $30 \pm 7 \mathrm{~kg} / \mathrm{m}^{2}$ showed that a low-flow $\mathrm{ECCO}_{2} \mathrm{R}$ device enabled very low tidal volume ventilation with moderate increase in $\mathrm{PaCO}_{2}$ in patients with mild-to-moderate ARDS. $E C C \mathrm{O}_{2} \mathrm{R}$ is promising in this setting in the obese population, to further avoid volutrauma using very low tidal volumes associated with high PEEP with the aim of avoiding closed alveoli and atelectasis.

\section{Research agenda}

Because obesity status is often an exclusion criterion in the major randomized controlled studies in ARDS, specific outcome studies are needed in obese patients with ARDS. Results from the main randomized controlled studies assessing effects of mechanical ventilation settings (optimal ventilatory mode, tidal volume, PEEP value, recruitment maneuver type and modalities) cannot be generalized to obese patients. Future randomized controlled trials are necessary to evaluate the effect of different strategies of ARDS management on outcomes in this population. Similar evaluations should be conducted in obese patients without ARDS at admission to the ICU with the aim of preventing development of ARDS. The most recent physiologic studies suggest that an individualized approach should be evaluated in obese patients with ARDS.

\section{Conclusion}

Obese patients have an increased risk of ARDS. Atelectasis formation, increased baseline oxygen consumption and iatrogenic ventilator management could explain the higher incidence of ARDS in obese patients. Lung volumes are not increased in obese patients compared to non-obese patients, and settings of protective ventilation should be applied, based on the ideal body weight and not on the real body weight. Transthoracic pressure is higher in obese patients than in non-obese patients, so driving pressure may be not appropriate to assess the severity of ARDS and to guide ventilatory management in obese patients. Monitoring of eesophageal pressure seems particularly interesting in morbidly obese patients, in order to assess the real transpulmonary pressure and set optimal PEEP levels. To counteract the onset of atelectasis in the dependent region of the lungs, prone positioning may be advised in obese patients with ARDS, by a trained team. Individualized treatment remains the best option for optimal outcomes, taking into account the pathophysiology of the obese patient.

\section{Acknowledgements}

None. 


\section{Funding}

Publication costs were funded by the University Hospital of Montpellier department of Research Clinic.

\section{Availability of data and materials}

Not applicable.

\section{Authors' contributions}

All authors contributed to the design and revision of the manuscript and read and approved the final version for publication.

\section{Ethics approval and consent to participate}

Not applicable.

\section{Consent for publication}

Not applicable.

\section{Competing interests}

The authors declare that they have no competing interests.

\section{Publisher's Note}

Springer Nature remains neutral with regard to jurisdictional claims in published maps and institutional affiliations.

\section{Published online: 09 March 2019}

\section{References}

1. Afshin A, Forouzanfar MH, Reitsma MB, et al. Health effects of overweight and obesity in 195 countries over 25 years. N Engl J Med. 2017;377:13-27.

2. Hales CM, Fryar CD, Carroll MD, et al. Differences in obesity prevalence by demographic characteristics and urbanization level among adults in the United States, 2013-2016. JAMA. 2018;319:2419-29.

3. Pepin JL, Timsit JF, Tamisier R, et al. Prevention and care of respiratory failure in obese patients. Lancet Respir Med. 2016;4:407-18.

4. Ball L, Serpa Neto A, Pelosi P. Obesity and survival in critically ill patients with acute respiratory distress syndrome: a paradox within the paradox. Crit Care. 2017:21:114.

5. De Jong A, Molinari N, Pouzeratte $Y$, et al. Difficult intubation in obese patients: incidence, risk factors, and complications in the operating theatre and in intensive care units. Br J Anaesth. 2015;114:297-306.

6. Gong MN, Bajwa EK, Thompson BT, Christiani DC. Body mass index is associated with the development of acute respiratory distress syndrome. Thorax. 2010;65:44-50.

7. Anzueto A, Frutos-Vivar F, Esteban A, et al. Influence of body mass index on outcome of the mechanically ventilated patients. Thorax. 2011;66:66-73.

8. De Jong A, Molinari N, Sebbane $M$, et al. Feasibility and effectiveness of prone position in morbidly obese patients with ARDS: a case-control clinical study. Chest. 2013;143:1554-61.

9. O'Brien JM Jr, Phillips GS, Ali NA, et al. Body mass index is independently associated with hospital mortality in mechanically ventilated adults with acute lung injury. Crit Care Med. 2006;34:738-44.

10. Zhi G, Xin W, Ying W, et al. "Obesity paradox" in acute respiratory distress syndrome: asystematic review and meta-analysis. PLoS One. 2016;11: e0163677.

11. Shashaty MG, Stapleton RD. Physiological and management implications of obesity in critical illness. Ann Am Thorac Soc. 2014;11:1286-97.

12. De Jong A, Chanques $\mathrm{G}$, Jaber S. Mechanical ventilation in obese ICU patients: from intubation to extubation. Crit Care. 2017;21:63.

13. Hibbert KRM, Malhotra A. Obesity and ARDS. Chest. 2012;142:785-90.

14. Gajic O, Dabbagh O, Park PK, et al. Early identification of patients at risk of acute lung injury: evaluation of lung injury prediction score in a multicenter cohort study. Am J Respir Crit Care Med. 2011;183:462-70.

15. Elie-Turenne MC, Hou PC, Mitani A, et al. Lung injury prediction score for the emergency department: first step towards prevention in patients at risk. Int J Emerg Med. 2012;5:33.

16. Kor DJ, Lingineni RK, Gajic O, et al. Predicting risk of postoperative lung injury in high-risk surgical patients: a multicenter cohort study. Anesthesiology. 2014;120(5):1168-81.

17. Levitt JE, Calfee CS, Goldstein BA, et al. Early acute lung injury: criteria for identifying lung injury prior to the need for positive pressure ventilation. Crit Care Med. 2013:41:1929-37.
18. O'Brien JM Jr, Welsh $\mathrm{CH}$, Fish RH, et al. Excess body weight is not independently associated with outcome in mechanically ventilated patients with acute lung injury. Ann Intern Med. 2004;140:338-45.

19. Morris AE, Stapleton RD, Rubenfeld GD, et al. The association between body mass index and clinical outcomes in acute lung injury. Chest. 2007;131:342-8.

20. Stapleton RD, Dixon AE, Parsons PE, et al. The association between BMl and plasma cytokine levels in patients with acute lung injury. Chest. 2010;138:568-77.

21. Soto GJ, Frank AJ, Christiani DC, Gong MN. Body mass index and acute kidney injury in the acute respiratory distress syndrome. Crit Care Med. 2012:40:2601-8.

22. De Jong A, Cossic J, Verzilli D, et al. Impact of the driving pressure on mortality in obese and non-obese ARDS patients: a retrospective study of 362 cases. Intensive Care Med. 2018;44:1106-14.

23. Ni YN, Luo J, Yu H, et al. Can body mass index predict clinical outcomes for patients with acute lung injury/acute respiratory distress syndrome? A meta-analysis. Crit Care. 2017;21:36.

24. Brown SM, Wilson E, Presson AP, et al. Predictors of 6-month health utility outcomes in survivors of acute respiratory distress syndrome. Thorax. 2017; 72:311-7.

25. Pelosi $P$, Croci M, Ravagnan I, et al. The effects of body mass on lung volumes, respiratory mechanics, and gas exchange during general anesthesia. Anesth Analg. 1998;87:654-60.

26. De Jong A, Carreira S, Na N, et al. Diaphragmatic function is enhanced in fatty and diabetic fatty rats. PLoS One. 2017;12:e0174043.

27. De Jong A, Verzilli $D$, Sebbane $M$, et al. Medical versus surgical ICU obese patient outcome: a propensity-matched analysis to resolve clinical trial controversies. Crit Care Med. 2018;46:e294-301.

28. Burns SM, Egloff MB, Ryan B, et al. Effect of body position on spontaneous respiratory rate and tidal volume in patients with obesity, abdominal distension and ascites. Am J Crit Care. 1994:3:102-6.

29. Jaber $\mathrm{S}$, Bellani $\mathrm{G}$, Blanch $\mathrm{L}$, et al. The intensive care medicine research agenda for airways, invasive and noninvasive mechanical ventilation. Intensive Care Med. 2017;43:1352-65.

30. O'Brien JM Jr, Philips GS, Ali NA, et al. The association between body mass index, processes of care, and outcomes from mechanical ventilation: a prospective cohort study. Crit Care Med. 2012:40:1456-63.

31. Bime C, Fiero M, Lu Z, et al. High positive end-expiratory pressure is associated with improved survival in obese patients with acute respiratory distress syndrome. Am J Med. 2017;130:207-13.

32. Pirrone $M$, Fisher $D$, Chipman $D$, et al. Recruitment maneuvers and positive end-expiratory pressure titration in morbidly obese ICU patients. Crit Care Med. 2016:44:300-7.

33. Nestler C, Simon P, Petroff D, et al. Individualized positive end-expiratory pressure in obese patients during general anaesthesia: a randomized controlled clinical trial using electrical impedance tomography. $\mathrm{Br} J$ Anaesth. 2017;119:1194-205.

34. Amato MB, Meade MO, Slutsky AS, et al. Driving pressure and survival in the acute respiratory distress syndrome. N Engl J Med. 2015;372:747-55.

35. Guerin C, Papazian L, Reignier J, et al. Effect of driving pressure on mortality in ARDS patients during lung protective mechanical ventilation in two randomized controlled trials. Crit Care. 2016:20:384.

36. Behazin N, Jones SB, Cohen Rl, Loring SH. Respiratory restriction and elevated pleural and esophageal pressures in morbid obesity. J Appl Physiol (1985). 2010;108:212-8.

37. Baedorf Kassis E, Loring SH, Talmor D. Mortality and pulmonary mechanics in relation to respiratory system and transpulmonary driving pressures in ARDS. Intensive Care Med. 2016:42:1206-13.

38. Eichler L, Truskowska K, Dupree A, Busch P, Goetz AE, Zöllner C. Intraoperative ventilation of morbidly obese patients guided by transpulmonary pressure. Obes Surg. 2018;28:122-9.

39. Fumagalli J, Berra L, Zhang $C$, et al. Transpulmonary pressure describes lung morphology during decremental positive end-expiratory pressure trials in obesity. Crit Care Med. 2017;45:1374-81.

40. Pandit JJ, Andrade J, Bogod DG, et al. 5th National Audit Project (NAP5) on accidental awareness during general anaesthesia: summary of main findings and risk factors. Br J Anaesth. 2014;113:549-59.

41. Guérin C, Reignier J, Richard JC, et al. Prone positioning in severe acute respiratory distress syndrome. N Engl J Med. 2013;368:2159-68.

42. Weig $T$, Janitza $S$, Zoller $M$, et al. Influence of abdominal obesity on multiorgan dysfunction and mortality in acute respiratory distress syndrome patients treated with prone positioning. J Crit Care. 2014;29:557-61. 
43. Combes A, Brodie D, Chen YS, et al. The ICM research agenda on extracorporeal life support. Intensive Care Med. 2017;43:1306-18.

44. Schmid C, Philipp A, Hilker M, et al. Venovenous extracorporeal membrane oxygenation for acute lung failure in adults. J Heart Lung Transplant. 2012;31:9-15.

45. Kon ZN, Dahi S, Evans CF, et al. Class III obesity is not a contraindication to venovenous extracorporeal membrane oxygenation support. Ann Thorac Surg. 2015;100:1855-60.

46. Salna M, Chicotka S, Biscotti M 3rd, et al. Morbid obesity is not a contraindication to transport on extracorporeal support. Eur J Cardiothorac Surg. 2018;53:793-8.

47. Combes A, Hajage D, Capellier G, et al. Extracorporeal membrane oxygenation for severe acute respiratory distress syndrome. N Engl J Med. 2018:378:1965-75

48. Swol J, Buchwald D, Dudda M, et al. Veno-venous extracorporeal membrane oxygenation in obese surgical patients with hypercapnic lung failure. Acta Anaesthesiol Scand. 2014;58:534-8.

49. Morelli A, Del Sorbo L, Pesenti A, et al. Extracorporeal carbon dioxide removal (ECCO2R) in patients with acute respiratory failure. Intensive Care Med. 2017:43:519-30.

50. Schmidt M, Jaber S, Zogheib E, et al. Feasibility and safety of low-flow extracorporeal $\mathrm{CO} 2$ removal managed with a renal replacement platform to enhance lung-protective ventilation of patients with mild-to-moderate ARDS. Crit Care. 2018;22:122. 